\title{
Meteor showers of cometary origin in the Solar System: Revised predictions ${ }^{\star}$
}

\author{
F. Selsis ${ }^{1}$, J. Brillet ${ }^{2}$, and M. Rapaport ${ }^{2}$ \\ 1 Centro de Astrobiología (INTA-CSIC), Ctra. de Ajalvir, km. 4, 28850, Torrejón de Ardoz, Madrid, Spain \\ 2 Observatoire de Bordeaux, BP 89, 33270, Floirac, France
}

Received 7 May 2003 / Accepted 17 November 2003

\begin{abstract}
For meteor shower forecasting on the other planets of the Solar System, we have identified a list of periodic comets having close enough encounters with planetary orbits to produce regular showers. We revisit a previous study, done with an analytical approximation of the problem that proves to be unsuitable for the present purpose. By applying an accurate numerical method, we found significant differences in the results and identified more cometary candidates from the same initial sample. We give approximate dates for the potential meteor showers, highlighting the ones expected to occur during future planetary missions.
\end{abstract}

Key words. comets: general - meteors, meteoroids

\section{Introduction}

One of the motivations for the study of meteors in the atmosphere of the Earth, especially during Leonid showers (see for instance Jenniskens 2002b), is related to the important role played by meteors in the chemistry of the Earth's early atmosphere. Indeed, the estimated flux of meteoroids to the primitive Earth 4 Gyrs ago is 200 times higher than today (Chyba $\&$ Sagan 1997) and was probably much higher earlier. As the present flux is $4 \times 10^{7} \mathrm{~kg} \mathrm{yr}^{-1}$ (Love \& Brownlee 1993), meteoroids have delivered a considerable amount of organic matter to the early Earth (Jenniskens 2001). The chemical interaction between the ablated content of the particles and the atmospheric compounds is not well known, nor is the modification in the chemical composition of the atmosphere induced by the heating itself. Observing meteors in our atmosphere gives us some information about such processes but only in the case of an $\mathrm{O}_{2}$-rich atmosphere while the primitive atmosphere was not oxidizing but more likely made of $\mathrm{N}_{2}, \mathrm{CO}_{2}$ and $\mathrm{H}_{2} \mathrm{O}$ (Kasting 1993) or mildly reducing, with a significant fraction of $\mathrm{CH}_{4}$ and $\mathrm{H}_{2}$ (Pavlov et al. 2000). Meteor showers in the atmosphere of Mars or Venus $\left(\mathrm{CO}_{2}\right.$-rich atmospheres) or in the reducing atmosphere of the giant planets or Titan would therefore provide conditions much closer to the ones prevailing on the early Earth. For example, the nitrogen oxide (NO) may have been involved in the formation of the first peptides (Commeyras et al. 2002). By heating the atmosphere of the present Earth to about $5000 \mathrm{~K}$, meteors efficiently produce NO.

\footnotetext{
Send offprint requests to: F. Selsis,

e-mail: selsis@obs.u-bordeaux1.fr

$\star$ Table 3 is only available in electronic form at http://www. edpsciences.org
}

However, this production of NO would be significantly different in a $\mathrm{CO}_{2}$-rich atmosphere.

The ability to predict the crossing of a cometary trail by a planet has also other interest. On Mercury, where no meteor occurs given the absence of an atmosphere, the impact of interplanetary dust plays an important role in the surface erosion. The gamma-ray and neutron spectrometers of the future ESA Bepi Colombo mission will study the erosion caused by the solar wind and micro-impacts. Being able to correlate a predicted increase of the bombardment with a qualitative change in the observations would allow to distinguish the spectral contribution of the micro-impacts.

Eventually, as incoming particles might damage spacecrafts, such predictions can help to protect them.

Accurate forecasting of meteor showers on planets, as was done successfully for showers on Earth by the Kazan research group (e.g. Kondrat'eva \& Reznikov 1985), and since 1999 by many authors for the Leonids, is a very complicated task. The first step is to identify cometary orbits that are close enough to a planetary orbit to deliver particles to the planet. The present paper is dedicated to this selection of "comet candidates" using cometary ephemerides. The next steps, not addressed here, would consist of describing precisely, for each comet selected, the ejection of particles and their trajectory influenced by radiation pressure and perturbations due to the planets, to determine the crossing point of individual trails and also the strength of the showers. For more details, see the papers dedicated to the forecasting of Leonid showers (Asher 1999; Lyytinen \& Van Flandern 2000; Vaubaillon 2002; Jenniskens 2002a).

In a previous study, Larson (2001, hereafter L01) identified a list of periodic comets having a close encounter with a planetary orbit and therefore susceptible to producing meteor 
Table 1. Validity of the L01 method. For each cometary trail approaching a planet at less than $5 R_{1}$ and selected by L01, we have calculated the ratio $r=D_{\mathrm{L} 01} / d$, where $d$ is the minimum separation obtained numerically and $D_{\mathrm{L} 01}$ the approximation of this value by L01 method. This table gives the statistic of $r$ and shows serious discrepancies except for the Earth for which the L01 method had been validated.

\begin{tabular}{lccccc}
\hline \hline Planet & $\begin{array}{c}\text { Mean value } \\
\text { of } r\end{array}$ & $\begin{array}{c}\text { Standard } \\
\text { deviation }\end{array}$ & $\begin{array}{c}\text { Max. } \\
\text { value }\end{array}$ & $\begin{array}{c}\text { Min. } \\
\text { value }\end{array}$ & $\begin{array}{c}\text { Number } \\
\text { of cases }\end{array}$ \\
\hline Venus & 29.48 & - & 29.48 & 29.48 & 1 \\
Earth & 1.015 & 0.016 & 1.037 & 1.001 & 4 \\
Mars & 2.110 & 1.391 & 3.094 & 1.127 & 2 \\
Jupiter & 2.928 & 2.644 & 16.778 & 1.000 & 233 \\
Saturn & 3.475 & 3.289 & 15.322 & 1.001 & 40 \\
Uranus & 5.843 & 4.643 & 14.784 & 1.824 & 10 \\
Neptune & 7.188 & 4.799 & 13.671 & 2.841 & 6 \\
\hline
\end{tabular}

showers. However, the analytical method used by L01 relied on a wrong hypothesis (see Method). Hence, the published list was not exhaustive and both dates and minimum distances need to be corrected. This the purpose of this paper.

\section{Method}

The criterion used in L01 to select the comets was the following: when the minimum distance between a cometary and a planetary orbit is lower or comparable to the Roche lobe radius of the planet $R_{1}$ then a residual cometary trail is likely to be intercepted by the planet, producing a meteor shower. The list given in L01 is divided in two parts: the encounters at less than $R_{1}$ and the ones between $R_{1}$ and $5 R_{1}$. The present study also adopts these criteria (we have used the same approximation as in L01 to compute $R_{1}$ ).

To calculate the minimal separation between orbits, an analytical method was deloped in L01, assuming that the length measured between orbits at the intersection of the two orbital planes is a robust approximation of the absolute minimum distance. As shown in Fig. 1, this assumption is not justified and the L01 method cannot be used as a general way to estimate the minimum separation. The efficiency of the L01 method was validated by its ability to retrieve the parent comets and the correct date of the main meteor showers observed on Earth (Leonids and Perseids). However, a comparison between our minimal distances of approaches $d$ obtained by our numerical method and those, $D_{\mathrm{L} 01}$, calculated by L01, shows serious discrepancies. Statistics of the ratio $r=D_{\mathrm{L} 01} / d$ is presented in Table 1. One can see that the Earth case is peculiar and that the analytical method proposed by L01 does not apply to the other planets. In the Earth case, the error on the minimum distance calculated by L01 never exceeds $4 \%$ for the 4 cases we considered. However this apparent good agreement for the Earth is a coincidence. We did test calculations for comets having larger minimum distances and found strong discrepancies between the numerical and the L01 method. For instance, the ratio $r$ is 6.8 for the comet $45 \mathrm{P} /$ Honda-Mrkos-Pajdusakova, 2.5 for $1 \mathrm{P} /$ Halley, 3.7 for $2 \mathrm{P} /$ Encke and even 12.8 for 107P/Wilson-Harrington.
The method we applied can be summarized as follow:

- We used the cometary orbital elements given by the JPL DASTCOM $^{1}$ database and the planetary orbital elements from (Standish et al. 1992). Sect. 2 in L01 provides a clear and detailed description of the orbits through their elements (semi-major axis, eccentricity, inclination, longitude of the ascending node and argument of perihelion).

- Two points on the planetary and cometary orbits may be located respectively by two vectors $\boldsymbol{r}_{\mathrm{p}}\left(\theta_{\mathrm{p}}\right)$ and $\boldsymbol{r}_{\mathrm{c}}\left(\theta_{\mathrm{c}}\right)$ having the same origin on the common focus of the ellipses. As usual, $\theta_{\mathrm{p}}$ and $\theta_{\mathrm{c}}$ are the angles (the true anomalies) between these vectors and the axes pointing towards the corresponding perihelions. The distance between these points is given by the Euclidian norm $D\left(\theta_{\mathrm{p}}, \theta_{\mathrm{c}}\right)=\left\|\boldsymbol{r}_{\mathrm{p}}\left(\theta_{\mathrm{p}}\right)-\boldsymbol{r}_{\mathrm{c}}\left(\theta_{\mathrm{c}}\right)\right\|$. Then, the planetary angles $\theta_{\text {min }}$ corresponding to the minimal distances $d$ are obtained by solving the set of equations

$$
\frac{\partial D}{\partial \theta_{\mathrm{p}}}=0, \quad \frac{\partial D}{\partial \theta_{\mathrm{c}}}=0 .
$$

The complete set of the solutions gives all the angles corresponding to the local minima and maxima of $D\left(\theta_{\mathrm{p}}, \theta_{\mathrm{c}}\right)$ as well as the saddle points. In the context of this study where only the cometary trails are concerned, the solutions of the cometary angles are not useful except for calculating $d$.

- Unfortunately, there is no possibility to obtain the solutions in a closed form and numerical methods are needed (see for example Murray et al. 1980). Also, the number of local minima cannot be deduced. In this situation, the first step is to isolate smallest rectangular domains of $\left(\theta_{\mathrm{p}}, \theta_{\mathrm{c}}\right)$ in the square $[0,2 \pi] \times[0,2 \pi]$ including a connected domain where $D\left(\theta_{\mathrm{p}}, \theta_{\mathrm{c}}\right) \leq k R_{1}$ with $k=5$ or $k=1$. This is obtained by calculating $D\left(\theta_{\mathrm{p}}, \theta_{\mathrm{c}}\right)$ on a grid. The mesh must be small enough to avoid missing narrow domains. For the selected planet-comet pairs, numerous cases have been found with two domains below $5 R_{1}$, a few cases with one domain below $R_{1}$ and another below $5 R_{1}$, no case with two domains below $R_{1}$ and no case with more than two approaches smaller than $5 R_{1}$.

- The second step is the determination, in each domain, of the planetary angle $\theta_{\text {min }}$ (and the associated cometary angle) for the minimal distance $d$. This has been achieved by two independent methods. The first uses the Newton-Raphson algorithm, the second an adaptative discretization of the selected region. Both methods yield the same results with a high accuracy. Some technical difficulties may arise during the computing process. For instance, two relative minima may be present in the same domain. Then, the minimum minimorum is always selected. Finally, $d$ values are calculated. We have also applied the L01 method to test its validity (see Table 1).

- The third step is the determination of the range $\left[\theta_{\text {in }}, \theta_{\text {out }}\right]$ of the planetary angles surrounding $\theta_{\min }$ where the distances satisfy the criterion $D\left(\theta_{\mathrm{p}}, \theta_{\mathrm{c}}\right) \leq k R_{1}$ with $k=5$ or $k=1$. These values are obtained by solving the set of equations

$$
\frac{\partial D}{\partial \theta_{\mathrm{c}}}=0, \quad D\left(\theta_{\mathrm{p}}, \theta_{\mathrm{c}}\right)=k R_{\mathrm{l}} \text {. }
$$

\footnotetext{
${ }^{1}$ http://ssd.jpl.nasa.gov/dastcom.html
} 


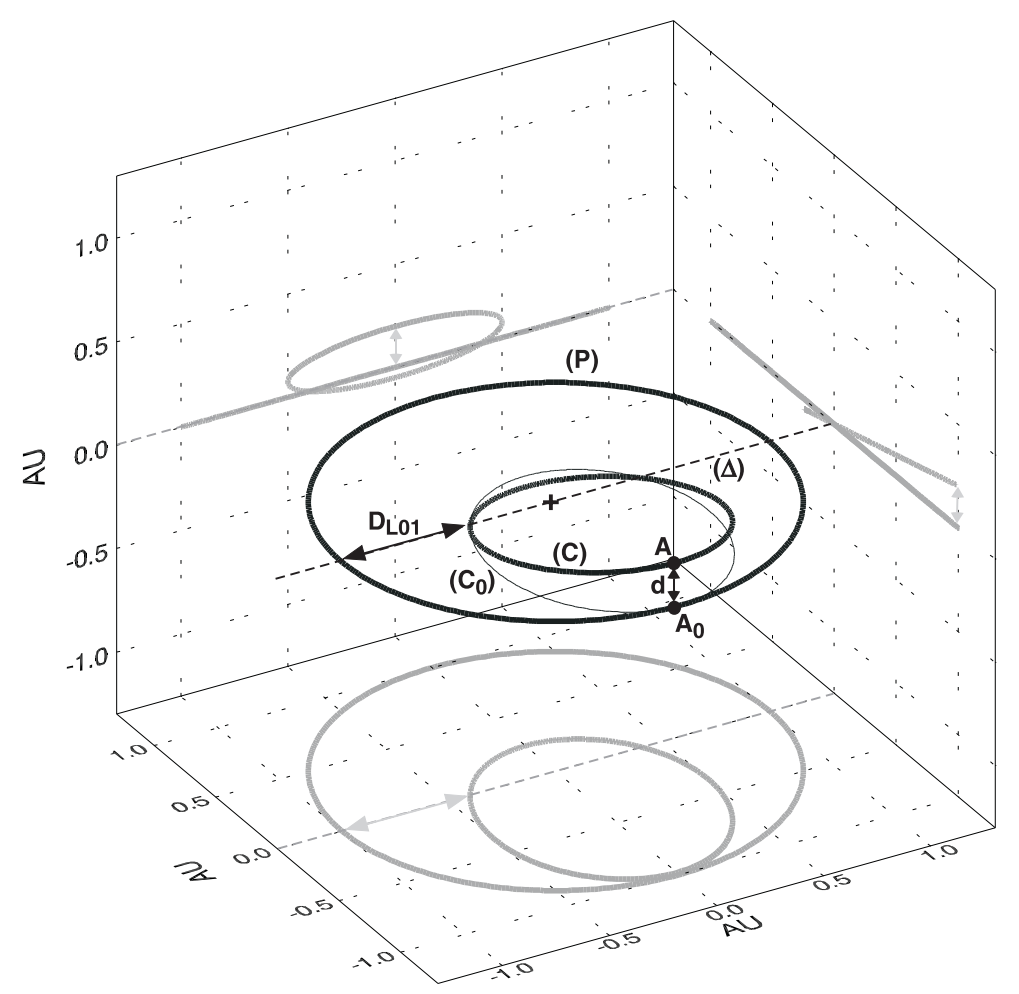

Fig. 1. A counter-example for the L01 method. This figure shows a schematic planetary orbit $(\mathrm{P})$ with an aphelion distance of 1 AU and a coplanar cometary orbit $\left(\mathrm{C}_{0}\right)$. Both have the same focus and a common aphelion $\mathrm{A}_{0}$. We define $(\Delta)$ as the axis perpendicular to the major axis of orbit $\left(\mathrm{C}_{0}\right)$ with the focus on it. Now, let us produce an orbit $(\mathrm{C})$ by rotating the orbit plane of $\left(\mathrm{C}_{0}\right)$ by a small angle $\phi$ around $(\Delta)$. According to L01, the smallest separation between orbits (P) and (C) should be $D_{\mathrm{L} 01}$. However, it is obvious here that, for low enough values of $\phi$, the distance $d$ between $\mathrm{A}$ and $\mathrm{A}_{0}$ is the smallest separation between orbits $(\mathrm{P})$ and $(\mathrm{C})$. Indeed, the value of $D_{\mathrm{L} 01}$ does not depend on $\phi$ while $d \approx \sin (\phi)$. Though the geometrical illustration given in L01 is rather convincing, this example demonstrates that the smallest distance between two ellipses with a same focus cannot be generally approximated by the distance measured at the intersection of the two orbital planes.

Here, only the Newton-Raphson algorithm has been used.

- Knowing $\theta_{\text {min }}, \theta_{\text {in }}, \theta_{\text {out }}$ and the period of the planet, we can convert these angular values in time or duration with the formula given in L01 (Keplerian approximation). With the dates of the last planetary perihelion passages, the $\theta_{\min }$ values are finally converted into dates.

\section{Results}

All the close encounters we found between the planets of the solar system and the comets listed in DASTCOM are given in Table 3.

\subsection{Mercury}

The initial purpose of this study was to search for possible particle showers on Mercury. Indeed, the surface erosion induced by micro-impacts and the possibility to observe particles produced by the bombardment with instruments on board the future mission BEPI COLUMBO (ESA) make the prediction of such showers very interesting. However we have not found a comet candidate (according to the criterion $d<5 R_{1}$ ) in this case. This is not surprising as there are only 4 comets listed in DASTCOM with a perihelion distance smaller than Mercury's aphelion distance $(0.466 \mathrm{AU})$. The closest encounter found is with comet $2 \mathrm{P} /$ Encke, with a minimum separation $0.026 \mathrm{AU}$, corresponding to 124 times the Roche lobe radius of Mercury. It is however interesting to note that $2 \mathrm{P} /$ Encke has the third smallest perihelion distance (0.33 AU) in DASTCOM and also a very short period (3.3 yrs). The high degassing activity due to these frequent and close perihelion passages is probably one of the reasons why $2 \mathrm{P} /$ Encke is responsible for regular faint meteor showers on Earth (the Taurids, in November) although the approach corresponding to this shower is not closer than $0.19 \mathrm{AU}$ (about $100 R_{1}$ ). The other reason is that this degassed material is highly dispersed due the perturbation of the stream by Jupiter (Babadzhanov 1998). It therefore may be relevant to consider the wide stream of $2 \mathrm{P} /$ Encke as a possible source of particle showers on Mercury. The predicted dates found during BEPI COLUMBO mission are 2009: 01/24, 04/22, 07/18, 10/14, 2010: 01/10, 04/08, 07/05, 10/01, 12/28, 2011: 03/26, 06/22, 09/18, 12/15, 2012: 03/12, 06/08, 09/04, 12/01.

\subsection{Venus}

In L01, no comet had been identified as a potential source of meteor showers on Venus. However, the comet 45P/Honda-Mrkos-Pajdusakova approaches the Venusian orbit at $0.0016 \mathrm{AU}\left(1.25 R_{1}\right)$. In this case, the minimum separation is 29 times smaller than the one calculated with the L01 method. This is the highest ratio we found (see Table 1). This comet 


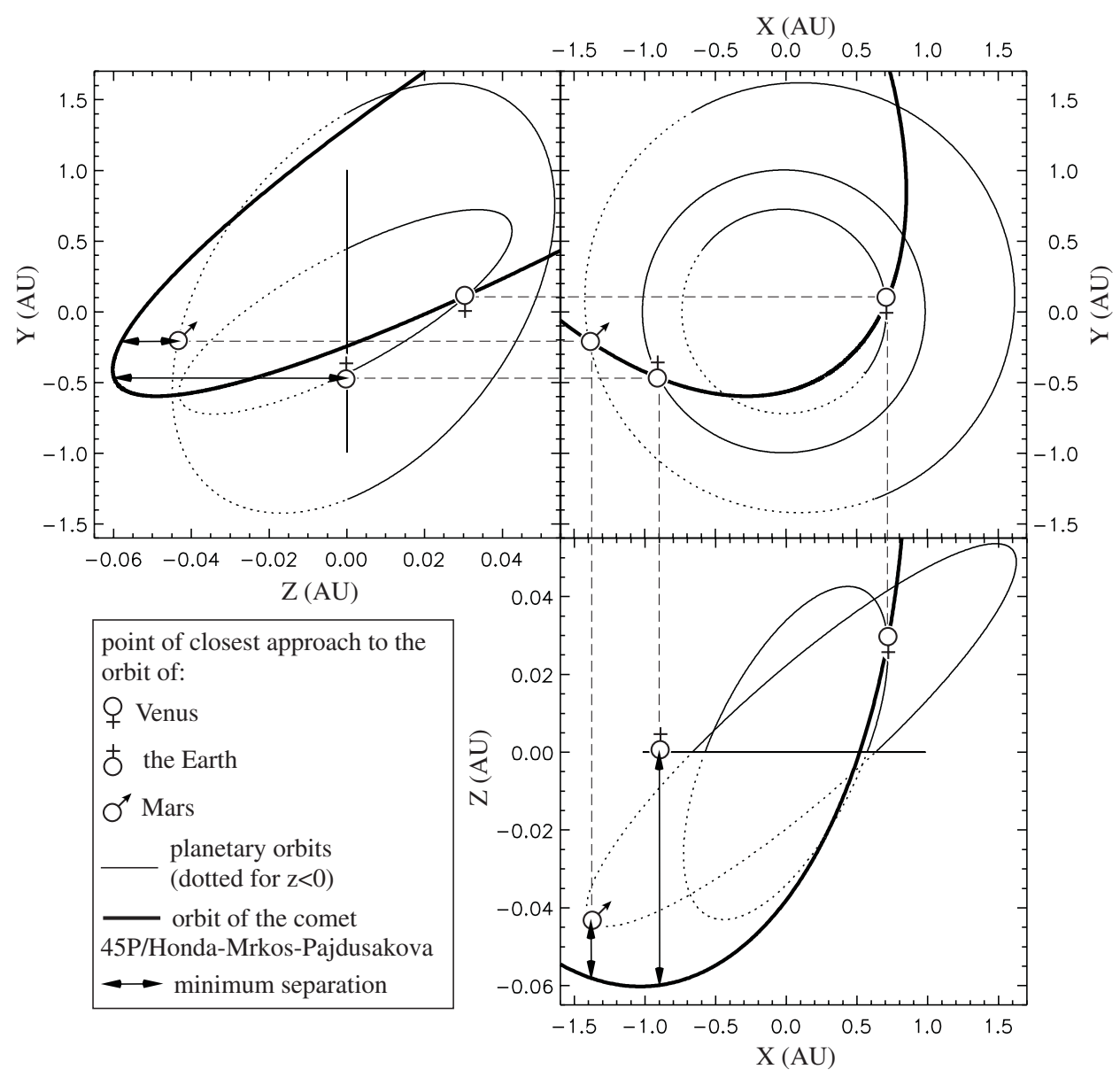

Fig. 2. Close encounters between comet 45P/Honda-Mrkos-Pajdusakova and Venus, the Earth and Mars. The projections of the orbits are shown in the coordinate system associated with the Earth ( $X Y$ being the plane of Earth's orbit). Note that the $Z$ scale is different to the $X$ and $Y$ scale. Dotted lines represent the orbit of Venus and Mars that are below the ecliptic $(Z<0)$. The comet approaches Venus' orbit at 0.0016 AU $\left(1.25 R_{\mathrm{l}}\right)$, Earth's orbit at $0.060 \mathrm{AU}\left(30 R_{\mathrm{l}}\right)$ and Mars' orbit at $0.015 \mathrm{AU}\left(13 R_{\mathrm{l}}\right)$. In the case of the Earth, the cometary trail is known to produce a faint shower (the alpha Capricornids) though the minimum separation is much bigger than our selection criterion. The comet is likely to cause meteor shower also on Venus and Mars for which the separation is smaller. Detailed modeling of this cometary stream is thus recommended.

has a period of about 5 years and is a very promising potential source of meteor showers. The Venus Express Mission might provide several observations of these events. Searching for encounters with a separation less than $10 R_{1}$ instead of $5 R_{1}$ does not provide more candidates.

A previous study by Beech (1998) also investigated possible meteor showers on Venus with a different selection criterion: the minimal distance was not calculated and the comets were chosen by the distance of their ascending or descending node to the Sun. When one of the nodal distances is equal to the semi-major axis of Venus' orbit at $\pm 0.1 \mathrm{AU}$, a close approach was assumed. Among the comets satisfying this condition, the author eventually kept two candidates, 27P/Crommelin and 7P/Pons-Winnecke, known to produce a meteor shower on Earth. However, 7P/Pons-Winnecke reached its perihelion in 2002, at a distance of 1.25 AU, whereas Beech found that the nodal distance criterion was satisfied for 7P/Pons-Winnecke's orbit, as it was during the 19th Century. New nodal distances are obviously greater than the perihelion distance and, thus, much farther from Venus' orbit. For 27/PCrommelin, we found a minimal separation of $20 R_{1}$ and we added this comet to the candidate list in Table 3.

Following Beech in this approach, we note that 45P/HondaMrkos-Pajdusakova is associated with the faint AlphaCapricornids shower, in August. The corresponding minimal separation between this comet and the orbit of the Earth is $0.060 \mathrm{AU}\left(30 R_{1}\right)$, which makes its close approach at $0.0016 \mathrm{AU}$ from Venus very promising (see Fig. 2).

\subsection{Earth}

For all the encounters at less than $5 R_{1}$ we obtained values very close to the ones found with the L01 method, with a difference always below 4\%. However, the comet C/1983 H1 (IRAS-Araki-Alcock), responsible for the faint Eta Lyrids shower (May 9) was not given in L01, for an unknown reason. Comets 109P/Swift-Tuttle, 55P/Tempel-Tuttle and 26P/Grigg-Skjellerup are respectively associated with the Perseids, Leonids and Pi Puppids meteor showers. A search with a criterion of $10 R_{1}$ gives two more candidates: $\mathrm{P} / 2000-\mathrm{G} 1$ 
Table 2. Extended sample for Mars: comets approaching the Martian orbit with a minimum separation between 5 and $10 R_{\mathrm{l}}$.

\begin{tabular}{lcccc}
\hline \hline Comet name & $\begin{array}{c}\text { Period } \\
\text { (year) }\end{array}$ & $\begin{array}{c}\text { Perihelion } \\
\text { distance }(\mathrm{AU})\end{array}$ & $\begin{array}{c}\text { Minimum } \\
\text { separation }\left(\mathrm{AU} / R_{\mathrm{I}}\right)\end{array}$ & $\begin{array}{c}\text { Next encounter } \\
(\mathrm{mm} / \mathrm{dd} / \mathrm{yyyy})\end{array}$ \\
\hline 114P/Wiseman-Skiff & 6.66 & 1.57 & $0.00625 / 5.66$ & $3 / 11 / 2004$ \\
9P/Tempel-1 & 5.51 & 1.50 & $0.00666 / 6.03$ & $2 / 27 / 2005$ \\
146P/Shoemaker-LINEAR & 7.88 & 1.32 & $0.00791 / 7.17$ & $1 / 10 / 2004$ \\
P/1999 D1 (Hermann) & 13.83 & 1.65 & $0.00861 / 7.81$ & $8 / 28 / 2004$ \\
C/1998 P1 (Williams) & $>10^{4}$ & 1.15 & $0.00989 / 8.97$ & $8 / 3 / 2004$ \\
\hline
\end{tabular}

$($ LINEAR $)($ period $=5.36 \mathrm{yrs}$, perihelion distance $=1.0 \mathrm{AU})$ and C/1999 J3 (LINEAR) (period $=62293$ yrs, perihelion distance $=0.98 \mathrm{AU}$ ) which are not associated with any observed increase in the terrestrial meteor activity. We found two cometary candidates, $\mathrm{P} / 2001 \mathrm{R} 1$ (LONEOS) and C/1998 U5 (LINEAR) with a minimum separation of $0.38 R_{1}$ and $1.75 R_{1}$ respectively. In both cases, the L01 method fails to give the minimum distance (giving respectively a value 3.09 and 1.13 times greater). The comet $\mathrm{P} / 2001 \mathrm{R}$ (LONEOS) was absent from L01's list because it was discovered later. This comet is the more interesting of the two due to the very close encounter it provides and its short period. Changing the selection criterion from $5 R_{1}$ to $10 R_{1}$ leads to 5 new cometary candidates that are given in Table 2. The four first ones have period and perihelion distances that may produce a dusty trail.

Christou \& Beurle (1999) selected potential parent comets of meteor showers on Mars by using a double criterion: a minimum separation smaller than $0.1 \mathrm{AU}$ and a relative velocity at Mars higher than $25 \mathrm{~km} \mathrm{~s}^{-1}$ in order to produce bright meteors. Two comets proved to satisfy this criterion: $1 \mathrm{P} /$ Halley $\left(P=76.0 \mathrm{yrs}, q=0.59 \mathrm{AU}, d=61 R_{1}\right)$ and $13 \mathrm{P} /$ Olbers $\left(P=69.6 \mathrm{yrs}, q=1.18 \mathrm{AU}, d=24 R_{\mathrm{l}}\right)$, where $q$ is the perihelion distance and $d$ the minimum separation. It should be noted that, according to this criterion, the comet C/1998 U5 (LINEAR), with a relative speed of $52 \mathrm{~km} \mathrm{~s}^{-1}$, should appear. Also, the comet 45P/Honda-Mrkos-Pajdusakova, already spotted in the case of Venus and responsible for a faint meteor shower on Earth, approaches Mars' orbit at $0.015 \mathrm{AU}\left(13 R_{1}\right)$, just above our extended threshold, with a relative speed of $23 \mathrm{~km} \mathrm{~s}^{-1}$, just below Christou and Beurle's speed criterion. Thus, 45P/Honda-Mrkos-Pajdusakova might well be the only known comet likely to produce meteor showers on all the terrestrial planets of the solar system having an atmosphere (see Fig. 2).

\subsection{Mars}

The relative speeds quoted above are from Treiman $\&$ Treiman (2000), a study also dedicated to the prediction of meteor showers on Mars. In their very detailed work, Treiman and Treiman gave a list of comets approaching Mars' orbit at less than $0.01 \mathrm{AU}\left(11 R_{1}\right)$ from Mars which is similar to our "extended" criterion of $10 R_{1}$ and, thus, we can compare their list of "nearest-Mars comet" with our. Three comets we found are not in their selection: P/2001 R1(LONEOS), discovered after the publication of their work, 146P/Shoemaker-LINEAR, for which better orbital elements are available since its perihelion passage in July 2000, and C/1998 P1 (Williams), probably ruled out because of its very long period. Then, some comets mistakenly appear in their list despite a minimum separation greater than $0.01 \mathrm{AU}: 104 \mathrm{P} /$ Kowal $2(d=0.079 \mathrm{AU})$, 104P/Kushida $(d=0.023 \mathrm{AU}), \mathrm{P} / 1998 \mathrm{~W} 1$ (Spahr) $(d=$ 0.14 AU). D/1884II (Barnard 1), D/1895II (Swift) and D/1984 W1 (Schoemaker 2), three D-comets (lost or no longer active) and also C/1985 II (Shoemaker), presented in their list are not included in DASTCOM and, therefore, were not considered in the present study.

The minimum separation is not the only parameter considered by Treiman and Treiman who have also taken into account the relative speed at Mars, possible future perturbations by Jupiter and available observations of dusty trails. They finally appointed $1 \mathrm{P} /$ Halley (despite the large minimum separation), 9P/Tempel-1 (because of its observed extended dusty trail), P/1999 D1 (Hermann) and C/1998 U5 (LINEAR) as the most promising candidates.

The comets 45P/Honda-Mrkos-Pajdusakova, 1P/Halley and 13P/Olbers, selected in these two previous studies but not by our selection criterion, have been included in the list given in Table 3.

\subsection{Jupiter and the outer planets}

There are significant differences between our list and the one given in L01. For instance, we found 48 comets approaching Jupiter's orbit at less than $R_{1}$ instead of 20 . We found also more comet candidates in the case of Saturn and Uranus and a few candidates for meteor showers on Neptune though none was found in the previous study. Some of the comets selected encounter a planetary orbit at two different points: for instance, Jupiter approaches the orbit of the comet 4P/Faye at less than $R_{1}$ between the 2233 rd and the 2292 nd days of its revolution, and has a second approach at less than $5 R_{1}$ between the 2709th and the 2932nd days. Such comets are marked with a flag and may appear twice in Table 3.

The comet $45 \mathrm{P} /$ Honda-Mrkos-Pajdusakova is again selected as it approaches Jupiter's orbit at less than $0.85 R_{\mathrm{l}}$.

The case of Saturn is made interesting by the presence of the Cassini probe after July 2004 and until July 2008 for its nominal mission. The comet $\mathrm{C} / 2001 \mathrm{~W} 2$ (Batters) could be a promising candidate because of its period (76 yrs) and its perihelion distance (1.05 AU) that can produce a dusty trail. The other encounters during Cassini's nominal mission are with orbits of comets having very long periods (>10000 yrs), except for the comet C/2001 S1 (Skiff) that has a shorter period 
(407 yrs) but a large perihelion distance (3.75 AU) implying a low activity and hence a low dust emission. In the case of an extended mission, the encounter in 2009 with the orbit of 126P/IRAS may motivate observations as the minimum separation is only $0.5 R_{1}$ and both period (13.3 yrs) and perihelion distance (1.7 AU) are favorable to produce a dusty trail.

Looking at Table 3, one can see that the delivery of cometary material, especially to Jupiter and Saturn, is continuous. Indeed, the meteor showers are overlapping because of the very long duration at passages at less than $R_{1}$ and, in other words, there is always a meteor shower there. It is thus difficult to talk about "showers", not only because of their duration, but because the low relative speed it implies is not favorable to produce bright meteors. For this reason, we focused on the closest approaches (within the Roche lobe radius) in Table 3, the exhaustive list being given for Jupiter only until 12/12/2004, and until the end of Cassini mission for Saturn. The complete list is given for Uranus and Neptune for which we identified fewer comets.

We did not find any cometary candidate for meteor showers on Pluto, according to the chosen criterion.

\section{Discussions}

\subsection{The selection criterion: $d<5 R_{\mathrm{I}}$}

This study is based on the selection criterion used by Larson (2001): comets approaching a planetary orbit at less than $R_{1}$ or a few times $R_{1}$ ( 5 was arbitrary chosen) can result in a regular meteor shower. This criterion works for the Earth in the sense that the strongest meteor showers can be found in this way but one should note that some comets known to produce regular showers on the Earth do not appear in the selected sample. As an example, the distance between the orbit of the comet Halley and the orbit of the Earth has 2 minima, one at $34 R_{1}$ corresponding to the Eta Aquarid shower, around May 8th, and one at nearly $80 R_{1}$ corresponding to the Orionid shower, around October 24th (Hughes 1987). However, the perihelion distance of this comet $(0.58 \mathrm{AU})$ is close enough to trigger the degassing of large amounts of material. The dense ejected stream is then spread by planetary perturbations, mainly due to Jupiter (McIntosh \& Jones 1988). The dense and dispersed stream is thus able to produce meteor showers even when the minimal separation with the planetary orbit is much greater than the Roche lobe radius of the planet. Among the known periodic comets with the closest perihelion one can also find $2 \mathrm{P} /$ Encke and 23P/Brorsen-Metcalf, responsible for faint showers despite the absence of very close encounters. Therefore, one could extend the search criterion, especially for comets having very close perihelion distances. In fact, previous studies often used a cutoff value of $0.1 \mathrm{AU}$ (McIntosh \& Jones 1988), similar, as pointed by Treiman and Treiman (2000), to the average dispersion of $0.08 \mathrm{AU}$ of meteor streams. In the present study we are mostly interested in strong showers that could be observed and we thus kept the criterion chosen by Larson, knowing that it could be too restrictive.

The DASTCOM database only contains comets for which reliable observations are available, allowing one to infer their orbital elements. It is known for instance that comet Thatcher (1861 I), one of the brightest comets of the 19th century, is associated with the Lyrids, but because of the lack of recent observations, it does not belong to DASTCOM and is therefore absent from our initial sample.

Other parameters should be considered after this preselection process, like the relative speed between the planet and the cometary stream, as done by Christou \& Beurle (1999) or Treiman \& Treiman (2000). Bright meteors might not be expected when the relative speed is too low, as on giant planets.

\subsection{Accuracy of the predictions}

The determination of relative positions of the orbits of planets and comets has been made considering Keplerian orbits; for the planets, we used the orbital elements given in the Explanatory Supplement to the Astronomical Almanac (Standish et al. 1992) and calculated the orbital positions using the classical formulae of the two body problem. The accuracy obtained is of course smaller than we could obtain by using integrated ephemerides such as the DE403 or DE405 ephemerides of JPL or those developed at IMCCE at Observatoire de Paris. (The accuracy of these integrated ephemerides is very high, at the level of $1000 \mathrm{~km}$ for the outer planets.)

An estimation of the precision of the positions obtained from Keplerian orbits is given in the Explanatory Supplement for the interval 1800-2050 AD. We again determined this precision comparing the positions obtained with those given by DE405. In the interval 2000-2020, the errors on the position of the planets are smaller than $10 \%$ of the Roche lobe radius, except for Mercury and Mars where this average ratio is about 20-30\%.

Concerning the comets, the determination of orbital elements from observation is affected by some uncertainties that vary from one comet to another. This uncertainty on the orbital distance is of the order of $10^{-7}-10^{-8} \mathrm{AU}$ for the best observations, and down to $10^{-4}-10^{-5} \mathrm{AU}$ for the poorly or very recently observed comets (P. Rocher, IMCCE, personal communication - see also the Cometary Notes ${ }^{2}$ ). It is interesting to note that the best data are obtained for the short period (repeated observations) and bright (easiest observations) comets which are precisely the ones that produce and sustain a dusty trail that may generate meteor showers. Due to the gravitational perturbation and non gravitational forces, this error on the position increases with time. We therefore restricted the determination of dates to the period before $12 / 31 / 2015$. Before this date, the error on the distance between the orbits remains negligible compared to the Roche lobe radius of the planets. The error on the determination of the epoch of the closest approaches is $<15 \mathrm{~min}$ for Mercury, Venus and the Earth, $<3 \mathrm{~h}$ for Mars, $<1$ day for Jupiter, $<4$ days for Saturn and $<3$ days for Uranus and Neptune. If more accurate predictions are needed, approximate ephemerides like the ones developed at IMCCE (Simon et al. 1994) could be used without problem.

\footnotetext{
2 http://www . imcce. fr/ephem/comets/HTML/english/ Comete_e.html
} 
This discussion concerns the internal errors consistency of our work and does not imply that the technique predicts meteor showers perfectly in practice. For that, more detailed models including non gravitational forces and planetary perturbations, and associated error estimates, would be needed.

\section{Conclusions}

Using the comet ephemerides given in the DASTCOM database, we have selected a list of periodic comets with close enough encounters with planetary orbits to produce regular meteor showers in the solar system. As a selection criterion, we used $d<5 R_{1}$, as suggested by Larson (2001: L01), where $d$ is the minimum separation between the cometary trails and the planets $\left(R_{1}\right)$ is the Roche lobe radius of the planet). Due to an incorrect geometrical argument in Larson's study, we have revised the calculations and obtained a significantly different sample of comets. The present list contains more cometary candidates and corrected encounter parameters (date and minimum orbital separation).

The present study has identified a possible shower on Venus associated with comet 45P/Honda-Mrkos-Pajdusakova and two on Mars (even more with an extended selection criterion). Using our results and those from Treiman \& Treiman (2000) we found that 45P/Honda-Mrkos-Pajdusakova, already associated with a faint shower on Earth, may well produce meteor showers also on Venus and Mars (Fig. 2) We have also found, in the case of Jupiter and Saturn, numerous "double encounters" with a comet trail during a single planetary revolution. The two giant planets prove to be always closer to a cometary stream than $d<5 R_{\mathrm{l}}$. This must result in a continuous delivery of material but probably not in bright showers, except for the closest passages, because of the low relative speeds.

Among the selected sample, we have highlighted the showers expected when planetary missions are scheduled. For these possible showers, we recommend more detailed studies (including models for the ejection of particles and non gravitational forces), similar to the one conducted to forecast the most recent Leonids showers on Earth (Asher 1999; Lyytinen \& Van Flandern 2000; Vaubaillon 2002; Jenniskens 2002a).

Acknowledgements. The authors wish to thank the referee, Dr D. Asher, for his thoughtful comments and suggestions that led to this final version.

\section{References}

Asher, D. J. 1999, MNRAS, 307, 919

Babadzhanov, P. B. 1998, Celest. Mech. Dynam. Astron., 69, 221

Beech, M. 1998, MNRAS, 294, 259

Christou, A. A., \& Beurle, K. 1999, Planet. Space Sci., 47, 1475

Chyba, C. F., \& Sagan, C. 1997, Comets and the origin and evolution of life, ed. P. J. Thomas, C. F. Chyba, \& C. P. McKay (SpringerVerlag), 147

Commeyras, A., Collet, H., Boiteau, L., et al. 2002, Polymer Int., 51, 661

Hughes, D. W. 1987, A\&A, 187, 879

Jenniskens, P. 2001, ESA SP-495, 495, 247

Jenniskens, P. 2002a, in Proc. ACM Berlin, 29 Jul.-02 Aug. 2002,

Jenniskens, P. 2002b, WGN, 30, 218

Kasting, J. F. 1993, Science, 259, 920

Kondrat'eva, E. D., \& Reznikov, E. A. 1985, Sol. Syst. Res., 19, 96

Larson, S. L. 2001, AJ, 121, 1722

Love, S. G., \& Brownlee, D. E. 1993, Science, 262, 550

Lyytinen, E. J., \& Van Flandern, T. 2000, Earth Moon Planets, 82, 149

McIntosh, B. A., \& Jones, J. 1988, MNRAS, 235, 673

Murray, C. D., Williams, I. P., \& Hughes, D. W. 1980, MNRAS, 190, 733

Pavlov, A. A., Kasting, J. F., Brown, L. L., Rages, K. A., \& Freedman, R. 2000, J. Geophys. Res., 105, 11981

Simon, J. L., Bretagnon, P., Chapront, J., et al. 1994, A\&A, 282, 663

Standish, E. M., Newhall, X. X., Williams, J. G., \& Yeomans, D. K. 1992, Explanatory Supplement to the Astronomical Almanac, ed. P. K. Seidelmann (University Science Books)

Treiman, A. H., \& Treiman, J. S. 2000, J. Geophys. Res., 105, 24571

Vaubaillon, J. 2002, WGN, 30, 144 


\section{Online Material}


Table 3. List of comet trails and planet close encounters. Columns 2 and 3 indicate the periods $P$ and the perihelion distances $q$ of the comets, which give an indication of the possible extent of the cometary dust (see text). Comets with an eccentricity greater than or equal to 1 are excluded. The two next columns give the true anomalies $\theta_{\min }$ of the planets and the associated dates for the minimal approaches. Taking accuracy into consideration, no date is given after 12/31/2015. Columns 6 and 7 give respectively the durations of the approaches at less than $5 R_{1}$ and $R_{1}$ when they occur $\left(R_{1}\right.$ is the planetary Roche radius). Negative and positive values indicate respectively the durations before and after the point of minimal approach. Column 8 gives the minimal distance $d$ of the approach. The last column gives the same but expressed in terms of $R_{1}$. In the first column, a $\bullet$ following the comet name emphasizes that the planet approaches twice the comet trail during a single revolution. In principle, therefore, a comet name may appear twice in the list. Some comets approaching a planetary orbit at more than $5 R_{1}$ have been included for specific reasons (see text): they are indicated by a $\uparrow$. Some comets identified as potential parent of meteor showers by other authors have been added to our selection: B98 refers to Beech (1998), C\&B to Christou \& Beurle (1999) and T\&T to Treiman \& Treiman (2000).

\begin{tabular}{|c|c|c|c|c|c|c|c|c|}
\hline Comet name & $\begin{array}{c}P \\
\text { (year) } \\
\end{array}$ & $\begin{array}{c}q \\
(\mathrm{AU}) \\
\end{array}$ & $\begin{array}{l}\theta_{\min } \\
(\mathrm{rad})\end{array}$ & $\begin{array}{c}\text { Date } \\
(\mathrm{mm} / \mathrm{dd} / \mathrm{yy})\end{array}$ & $\begin{array}{c}\text { Dur. }<5 R_{1} \\
\quad \text { (day) }\end{array}$ & $\begin{array}{c}\text { Dur. }<R_{l} \\
\text { (day) }\end{array}$ & $\begin{array}{c}d \\
(\mathrm{AU}) \\
\end{array}$ & $d / R_{\mathrm{l}}$ \\
\hline \multicolumn{9}{|c|}{ Mercury } \\
\hline \multicolumn{9}{|c|}{ Year 2004 only } \\
\hline $2 \mathrm{P} /$ Encke $\dagger$ & 3.3 & 0.34 & 0.9603 & $1 / 3 / 2004$ & & & 0.0263 & 125.7 \\
\hline $2 \mathrm{P} /$ Encke $\dagger$ & 3.3 & 0.34 & 0.9603 & $3 / 31 / 2004$ & & & 0.0263 & 125.7 \\
\hline $2 \mathrm{P} /$ Encke $\dagger$ & 3.3 & 0.34 & 0.9603 & $6 / 27 / 2004$ & & & 0.0263 & 125.7 \\
\hline $2 \mathrm{P} /$ Encke $\dagger$ & 3.3 & 0.34 & 0.9603 & 9/23/2004 & & & 0.0263 & 125.7 \\
\hline $2 \mathrm{P} /$ Encke $\dagger$ & 3.3 & 0.34 & 0.9603 & $12 / 20 / 2004$ & & & 0.0263 & 125.7 \\
\hline \multirow{2}{*}{\multicolumn{9}{|c|}{$\begin{array}{c}\text { Venus } \\
\text { Until } 10 / 1 / 2007 \text { only }\end{array}$}} \\
\hline & & & & & & & & \\
\hline 45P/Honda-Mrkos-Pajdusakova & 5.2 & 0.53 & 6.0940 & $8 / 3 / 03$ & $-0.6,0.6$ & & 0.0016 & 1.251 \\
\hline 27P/Crommelin [B98] & 27.4 & 0.73 & 5.1765 & $2 / 14 / 04$ & & & 0.0255 & 19.98 \\
\hline 45P/Honda-Mrkos-Pajdusakova & 5.2 & 0.53 & 6.0940 & $3 / 15 / 04$ & $-0.6,0.6$ & & 0.0016 & 1.251 \\
\hline 27P/Crommelin [B98] & 27.4 & 0.73 & 5.1765 & $9 / 25 / 04$ & & & 0.0255 & 19.98 \\
\hline 45P/Honda-Mrkos-Pajdusakova & 5.2 & 0.53 & 6.0940 & $10 / 25 / 04$ & $-0.6,0.6$ & & 0.0016 & 1.251 \\
\hline 27P/Crommelin [B98] & 27.4 & 0.73 & 5.1765 & $5 / 8 / 05$ & & & 0.0255 & 19.98 \\
\hline 45P/Honda-Mrkos-Pajdusakova & 5.2 & 0.53 & 6.0940 & $6 / 7 / 05$ & $-0.6,0.6$ & & 0.0016 & 1.251 \\
\hline 27P/Crommelin [B98] & 27.4 & 0.73 & 5.1765 & $12 / 19 / 05$ & & & 0.0255 & 19.98 \\
\hline 45P/Honda-Mrkos-Pajdusakova & 5.2 & 0.53 & 6.0940 & $1 / 18 / 06$ & $-0.6,0.6$ & & 0.0016 & 1.251 \\
\hline \multicolumn{9}{|l|}{ April 2006: Arrival of Venus Express } \\
\hline 27P/Crommelin [B98] & 27.4 & 0.73 & 5.1765 & $8 / 1 / 06$ & & & 0.0255 & 19.98 \\
\hline 45P/Honda-Mrkos-Pajdusakova & 5.2 & 0.53 & 6.0940 & $8 / 31 / 06$ & $-0.6,0.6$ & & 0.0016 & 1.251 \\
\hline 27P/Crommelin [B98] & 27.4 & 0.73 & 5.1765 & $3 / 13 / 07$ & & & 0.0255 & 19.98 \\
\hline 45P/Honda-Mrkos-Pajdusakova & 5.2 & 0.53 & 6.0940 & $4 / 12 / 07$ & $-0.6,0.6$ & & 0.0016 & 1.251 \\
\hline \multicolumn{9}{|c|}{ Sept. 2007: End of Venus Express mission } \\
\hline \multicolumn{9}{|c|}{ 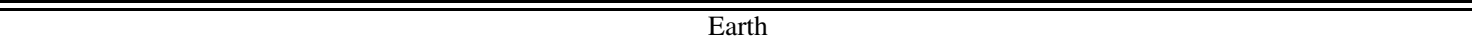 } \\
\hline \multicolumn{9}{|c|}{ Year 2003 only } \\
\hline 26P/Grigg-Skjellerup & 5.1 & 1.00 & 1.9254 & $4 / 23 / 03$ & $-0.7,0.7$ & & 0.0087 & 4.515 \\
\hline C/1983 H1 (IRAS-Araki-Alcock) & 959.1 & 0.99 & 2.2015 & $5 / 10 / 03$ & $-0.5,0.5$ & & 0.0058 & 2.993 \\
\hline 109P/Swift-Tuttle & 135.0 & 0.96 & 3.7785 & $8 / 12 / 03$ & $-0.6,0.6$ & $-0.1,0.1$ & 0.0009 & 0.489 \\
\hline 55P/Tempel-Tuttle & 33.2 & 0.98 & 5.4466 & $11 / 17 / 03$ & $-1.1,1.1$ & & 0.0079 & 4.093 \\
\hline \multicolumn{9}{|c|}{ Mars } \\
\hline P/2001 R1 (LONEOS) & 6.5 & 1.36 & 0.9492 & $11 / 24 / 03$ & $-1.3,1.3$ & $-0.2,0.2$ & 0.0004 & 0.382 \\
\hline \multicolumn{9}{|l|}{ Dec. 2003: Arrival of Mars Express } \\
\hline \multicolumn{9}{|c|}{ Jan. 2004: Arrival of the first Mars Exploration Rover (MER 2003) } \\
\hline \multicolumn{9}{|c|}{ Jan. 2004: Arrival of Nozumi mission } \\
\hline 1P/Halley [C\&B] & 76.0 & 0.59 & 1.2643 & $1 / 1 / 04$ & & & 0.0670 & 60.69 \\
\hline 146P/Shoemaker-LINEAR $\dagger$ & 7.9 & 1.32 & 1.3438 & $1 / 10 / 04$ & & & 0.0079 & 7.168 \\
\hline C/1998 U5 (LINEAR) & $>10^{3}$ & 1.24 & 1.5737 & $1 / 27 / 04$ & $-0.5,0.5$ & & 0.0019 & 1.749 \\
\hline 114P/Wiseman-Skiff $\dagger$ & 6.7 & 1.57 & 1.8902 & 3/11/04 & & & 0.0062 & 5.661 \\
\hline 13P/Olbers $[\mathrm{C} \& \mathrm{~B}]$ & 69.5 & 1.18 & 1.8941 & $3 / 11 / 04$ & & & 0.0265 & 24.01 \\
\hline \multicolumn{9}{|l|}{ April. 2004: End of MER 2003 mission } \\
\hline P/1999 D1 (Hermann) $\dagger$ & 13.8 & 1.65 & 3.2465 & $8 / 28 / 04$ & & & 0.0086 & 7.807 \\
\hline 9P/Tempel-1 $\dagger$ & 5.5 & 1.50 & 4.6982 & $2 / 27 / 05$ & & & 0.0067 & 6.033 \\
\hline 45P/Honda-Mrkos-Pajdusakova [T\&T] & 5.2 & 0.53 & 5.6761 & $5 / 25 / 05$ & & & 0.0147 & 13.31 \\
\hline P/2001 R1 (LONEOS) & 6.5 & 1.36 & 0.9492 & $10 / 11 / 05$ & $-1.3,1.3$ & $-0.2,0.2$ & 0.0004 & 0.382 \\
\hline \multicolumn{9}{|l|}{ Oct. 2005: End of Mars Express mission } \\
\hline 1P/Halley [C\&B] & 76.0 & 0.59 & 1.2643 & $11 / 18 / 05$ & & & 0.0670 & 60.69 \\
\hline 146P/Shoemaker-LINEAR $\dagger$ & 7.9 & 1.32 & 1.3438 & $11 / 27 / 05$ & & & 0.0079 & 7.168 \\
\hline C/1998 U5 (LINEAR) & $>10^{3}$ & 1.24 & 1.5737 & $12 / 14 / 05$ & $-0.5,0.5$ & & 0.0019 & 1.749 \\
\hline 114P/Wiseman-Skiff $\dagger$ & 6.7 & 1.57 & 1.8902 & $1 / 27 / 06$ & & & 0.0062 & 5.661 \\
\hline 13P/Olbers [C\&B] & 69.5 & 1.18 & 1.8941 & $1 / 27 / 06$ & & & 0.0265 & 24.01 \\
\hline Jan. 2006: End of Nozumi mission & & & & & & & & \\
\hline
\end{tabular}


F. Selsis et al.: Meteor showers in the solar system, Online Material $p 3$

Table 3. continued.

\begin{tabular}{|c|c|c|c|c|c|c|c|c|}
\hline \multicolumn{9}{|c|}{ Jupiter } \\
\hline \multicolumn{9}{|c|}{ Only cases with a minimal separation $<R_{1}$ ) are given } \\
\hline 42P/Neujmin $3 \bullet$ & 10.6 & 2.00 & 2.5796 & $2 / 10 / 04$ & $-140.2,152.5$ & $-28.1,28.5$ & 0.0297 & 0.234 \\
\hline P/1998 S1 (LINEAR-Mueller) & 9.1 & 2.55 & 2.7118 & $5 / 20 / 04$ & $-235.9,260.7$ & $-43.6,44.2$ & 0.0497 & 0.392 \\
\hline P/1998 W2 (Hergenrother) & 6.9 & 1.42 & 2.7653 & $6 / 29 / 04$ & $-164.9,190.7$ & $-33.5,34.3$ & 0.0111 & 0.088 \\
\hline 54P/de Vico-Swift-NEAT & 7.3 & 2.14 & 2.7667 & $6 / 30 / 04$ & $-360.3,487.2$ & $-56.8,65.0$ & 0.1029 & 0.811 \\
\hline 47P/Ashbrook-Jackson & 7.5 & 2.31 & 2.8053 & $7 / 29 / 04$ & $-362.0,315.1$ & $-37.7,36.9$ & 0.1078 & 0.850 \\
\hline P/2002 Q1 (Van Ness) & 6.6 & 1.52 & 2.8093 & $8 / 1 / 04$ & $-149.2,150.7$ & $-18.1,18.1$ & 0.1017 & 0.802 \\
\hline P/1983 V1 (Hartley-IRAS) & 21.5 & 1.28 & 2.9108 & $10 / 17 / 04$ & $-87.4,87.9$ & $-9.9,9.9$ & 0.1051 & 0.829 \\
\hline 85P/Boethin • & 11.2 & 1.11 & 2.9408 & $11 / 8 / 04$ & $-117.5,109.1$ & $-15.4,15.3$ & 0.0938 & 0.740 \\
\hline 97P/Metcalf-Brewington & 10.5 & 2.61 & 3.0322 & $1 / 17 / 05$ & $-177.1,166.5$ & $-28.2,27.9$ & 0.0726 & 0.573 \\
\hline 132P/Helin-Roman-Alu $2 \bullet$ & 8.2 & 1.91 & 3.0610 & $2 / 8 / 05$ & $-196.9,224.2$ & $-39.5,40.2$ & 0.0131 & 0.103 \\
\hline 16P/Brooks 2 & 6.9 & 1.83 & 3.1115 & $3 / 18 / 05$ & $-369.0,409.8$ & $-89.0,100.3$ & 0.0735 & 0.579 \\
\hline 4P/Faye • & 7.5 & 1.66 & 3.2683 & $7 / 15 / 05$ & $-188.7,265.3$ & $-28.9,29.7$ & 0.0861 & 0.679 \\
\hline 15P/Finlay $\bullet$ & 6.8 & 1.03 & 3.2975 & $8 / 6 / 05$ & $-200.0,153.7$ & $-32.0,31.2$ & 0.0112 & 0.089 \\
\hline 14P/Wolf & 8.2 & 2.41 & 3.3526 & $9 / 17 / 05$ & $-178.3,175.2$ & $-33.5,33.4$ & 0.0376 & 0.297 \\
\hline P/1991 V1 (Shoemaker-Levy 6) & 7.5 & 1.13 & 3.4951 & $1 / 2 / 06$ & $-136.0,136.9$ & $-26.2,26.2$ & 0.0306 & 0.242 \\
\hline 78P/Gehrels 2 & 7.2 & 2.00 & 3.5797 & $3 / 7 / 06$ & $-365.8,487.3$ & $-105.4,163.1$ & 0.0233 & 0.184 \\
\hline 72P/Denning-Fujikawa • & 9.0 & 0.78 & 3.6088 & $3 / 29 / 06$ & $-105.7,112.1$ & $-12.0,12.0$ & 0.1063 & 0.838 \\
\hline 67P/Churyumov-Gerasimenko & 6.6 & 1.29 & 3.7082 & $6 / 11 / 06$ & $-182.6,567.4$ & $-35.4,38.1$ & 0.0563 & 0.444 \\
\hline P/1990 V1 (Shoemaker-Levy 1) & 17.3 & 1.52 & 3.7920 & $8 / 13 / 06$ & $-105.5,113.9$ & $-14.3,14.4$ & 0.0965 & 0.761 \\
\hline 137P/Shoemaker-Levy $2 \bullet$ & 9.4 & 1.87 & 3.8567 & $9 / 30 / 06$ & $-161.0,167.9$ & $-25.3,25.5$ & 0.0801 & 0.632 \\
\hline 45P/Honda-Mrkos-Pajdusakova & 5.2 & 0.53 & 3.9597 & $12 / 15 / 06$ & $-300.1,154.3$ & $-23.6,20.1$ & 0.1077 & 0.849 \\
\hline P/1997 V1 (Larsen) & 10.9 & 3.29 & 3.9726 & $12 / 24 / 06$ & $-240.6,252.1$ & $-32.3,32.5$ & 0.0948 & 0.748 \\
\hline 18D/Perrine-Mrkos & 6.7 & 1.27 & 4.0100 & $1 / 21 / 07$ & $-208.4,163.5$ & $-32.5,31.5$ & 0.0457 & 0.360 \\
\hline 104P/Kowal 2 & 6.2 & 1.40 & 4.1129 & $4 / 6 / 07$ & $-200.7,286.8$ & $-35.3,37.9$ & 0.0789 & 0.622 \\
\hline 144P/Kushida • & 7.6 & 1.43 & 4.1917 & $6 / 2 / 07$ & $-148.7,152.3$ & $-29.4,29.5$ & 0.0096 & 0.076 \\
\hline $43 \mathrm{P} / \mathrm{Wc}$ & 6.5 & 1.58 & 4.2138 & $6 / 18 / 07$ & $-208.9,262.7$ & $-45.2,48.1$ & 0.0123 & 0.097 \\
\hline 148P/Anderson-LINEAR & 7.1 & 1.69 & 4.2324 & $7 / 1 / 07$ & $-201.6,731.0$ & $-42.3,45.8$ & 0.0265 & 0.209 \\
\hline P/1999 XB69 (LINEAR) & 9.4 & 1.64 & 4.2461 & $7 / 11 / 07$ & $-133.1,124.4$ & $-25.7,25.3$ & 0.0119 & 0.094 \\
\hline 139P/Vaisala-Oterma & 9.5 & 3.38 & 4.3250 & 9/5/07 & $-1203.0,495.3$ & $-131.2,107.4$ & 0.0405 & 0.319 \\
\hline 76P/West-Kohoutek-Ikemura & 6.5 & 1.60 & 4.3382 & $9 / 15 / 07$ & $-171.8,178.5$ & $-31.5,31.8$ & 0.0553 & 0.436 \\
\hline $75 \mathrm{P} / \mathrm{K}$ & 6.7 & 1.78 & 4.4987 & $1 / 6 / 08$ & $-421.2,333.5$ & $-146.6,97.6$ & 0.0202 & 0.159 \\
\hline 153P/Ikeya-Zhang & 366.5 & 0.51 & 4.5033 & $1 / 9 / 08$ & $-89.0,85.9$ & $-17.4,17.3$ & 0.0130 & 0.102 \\
\hline C/1997 J1 (Mueller) & $>10^{3}$ & 2.30 & 4.5658 & $2 / 22 / 08$ & $-89.9,86.3$ & $-10.1,10.1$ & 0.1045 & 0.824 \\
\hline 62P/Tsuchinshan 1 & 6.6 & 1.50 & 4.6398 & $4 / 14 / 08$ & $-173.2,540.1$ & $-31.1,32.5$ & 0.0668 & 0.527 \\
\hline P/1998 W1 (Spahr) & 6.6 & 1.73 & 4.6592 & $4 / 27 / 08$ & $-238.5,201.1$ & $-42.8,41.3$ & 0.0270 & 0.213 \\
\hline C/1995 O1 (Hale-Bopp) & $>10^{3}$ & 0.91 & 4.6726 & $5 / 6 / 08$ & $-84.3,84.2$ & $-16.8,16.8$ & 0.0084 & 0.066 \\
\hline 60P/Tsuchinshan 2 & 6.8 & 1.77 & 4.8521 & $9 / 6 / 08$ & $-200.4,580.9$ & $-34.5,36.8$ & 0.0783 & 0.618 \\
\hline P/2001 TU80 (LINEAR-NEAT) & 7.0 & 1.93 & 5.0212 & $12 / 29 / 08$ & $-615.5,269.4$ & $-51.5,42.5$ & 0.1000 & 0.788 \\
\hline 31P/Schwassmann-Wachmann 2 & 8.7 & 3.41 & 5.1220 & $3 / 6 / 09$ & $-537.5,821.7$ & $-130.5,197.3$ & 0.0596 & 0.470 \\
\hline 59P/Kearns-Kwee & 9.4 & 2.34 & 5.1220 & $3 / 6 / 09$ & $-182.7,187.1$ & $-36.0,36.2$ & 0.0179 & 0.141 \\
\hline 40P/Vaisala 1 & 10.8 & 1.78 & 5.2348 & $5 / 19 / 09$ & $-125.3,115.2$ & $-19.8,19.5$ & 0.0732 & 0.577 \\
\hline 149P/Mueller 4 & 9.0 & 2.65 & 5.4229 & $9 / 18 / 09$ & $-138.6,133.9$ & $-11.3,11.3$ & 0.1157 & 0.912 \\
\hline 52P/Harrington-Abell & 7.5 & 1.76 & 5.4799 & $10 / 25 / 09$ & $-184.2,168.6$ & $-31.2,30.9$ & 0.0533 & 0.420 \\
\hline 81P/Wild 2 & 6.4 & 1.59 & 5.6612 & $2 / 18 / 10$ & $-193.5,616.5$ & $-42.4,47.6$ & 0.0133 & 0.105 \\
\hline $\mathrm{P} / 2000 \mathrm{~B} 3($ & 8.0 & 1.70 & 5.6747 & $2 / 27 / 10$ & $-147.5,148.5$ & $-11.5,11.6$ & 0.1169 & 0.921 \\
\hline P/2000 Y3 (Scotti) & 11.3 & 4.05 & 5.7542 & $4 / 18 / 10$ & $-499.4,484.5$ & $-75.5,75.6$ & 0.0686 & 0.541 \\
\hline 124P/Mrkos & 5.7 & 1.47 & 6.0146 & $9 / 29 / 10$ & $-148.6,153.7$ & $-29.6,29.8$ & 0.0226 & 0.178 \\
\hline 26P/Grigg-Sl & 5.1 & 1.00 & 0.3677 & $11 / 1 / 11$ & $-201.1,170.8$ & $-36.7,34.7$ & 0.0536 & 0.423 \\
\hline 135P/Shoemaker-Levy 8 & 7.5 & 2.72 & 0.5510 & $2 / 25 / 12$ & $-407.2,488.1$ & $-81.5,90.0$ & 0.0793 & 0.626 \\
\hline 83P/Russell 1 & 6.1 & 1.61 & 0.6750 & $5 / 14 / 12$ & $-208.9,192.0$ & $-36.8,36.1$ & 0.0584 & 0.460 \\
\hline 34D/Gale & 11.0 & 1.18 & 0.7485 & $6 / 30 / 12$ & $-110.8,102.9$ & $-11.1,11.0$ & 0.1089 & 0.858 \\
\hline 117P/Helin-Roman-Alu 1 & 9.4 & 3.61 & 0.8977 & $10 / 4 / 12$ & $-434.3,621.3$ & $-90.1,95.9$ & 0.0164 & 0.130 \\
\hline 86P/Wild 3 & 6.9 & 2.31 & 0.9619 & $11 / 15 / 12$ & $-300.3,286.6$ & $-41.9,41.6$ & 0.0932 & 0.734 \\
\hline 143P/Kowal-Mrkos • & 8.9 & 2.55 & 0.9812 & $11 / 27 / 12$ & $-200.2,195.4$ & $-38.3,38.2$ & 0.0177 & 0.140 \\
\hline 91P/Russell 3 & 7.5 & 2.51 & 1.0011 & $12 / 10 / 12$ & $-333.1,259.7$ & $-54.5,51.7$ & 0.0480 & 0.378 \\
\hline 22P/Kopff & 6.5 & 1.58 & 1.7561 & $5 / 4 / 14$ & $-552.1,226.3$ & $-54.7,44.6$ & 0.0738 & 0.582 \\
\hline
\end{tabular}


Table 3. continued.

\begin{tabular}{|c|c|c|c|c|c|c|c|c|}
\hline \multicolumn{9}{|c|}{$\begin{array}{c}\text { The complete list (minimal separation }<5 R_{1} \text { ) is given until } 8 / 1 / 2008 \text { only } \\
\text { A reduced list (minimal separation }<R_{1} \text { ) is given after this date }\end{array}$} \\
\hline P/1994 J3 (Shoemaker 4) & 14.5 & 2.94 & 0.0093 & $7 / 21 / 03$ & $-229.8,247.8$ & & 0.2897 & 2.118 \\
\hline C/1998 M4 (LINEAR) & $>10^{3}$ & 2.60 & 0.1358 & $2 / 1 / 04$ & $-96.2,93.6$ & & 0.4854 & 3.548 \\
\hline \multicolumn{9}{|c|}{ July 2004: Arrival of Cassini mission } \\
\hline C/2001 W2 (BATTERS) & 75.9 & 1.05 & 0.3845 & $2 / 18 / 05$ & $-92.3,91.6$ & & 0.4382 & 3.204 \\
\hline C/2001 U6 (LINEAR) & $>10^{3}$ & 4.41 & 0.4097 & $3 / 29 / 05$ & $-40.6,40.5$ & & 0.6453 & 4.718 \\
\hline C/1997 BA6 (Spacewatch) & $>10^{3}$ & 3.44 & 0.7925 & $11 / 17 / 06$ & $-104.7,105.7$ & & 0.3103 & 2.269 \\
\hline C/2001 S1 (Skiff) & 407.6 & 3.75 & 0.9952 & 10/7/07 & $-119.3,115.6$ & & 0.3721 & 2.720 \\
\hline C/1998 T1 (LINEAR) & $>10^{3}$ & 1.47 & 1.0704 & $2 / 5 / 08$ & $-115.3,118.5$ & & 0.2716 & 1.986 \\
\hline \multicolumn{9}{|l|}{ July 2008: End of Cassini mission } \\
\hline C/1998 Q1 (LINEAR) & $>10^{3}$ & 1.58 & 1.2204 & 10/7/08 & $-124.8,128.2$ & $-24.0,24.2$ & 0.0421 & 0.307 \\
\hline 126P/IRAS & 13.3 & 1.70 & 1.4524 & $10 / 28 / 09$ & $-166.3,165.4$ & $-28.3,28.3$ & 0.0721 & 0.527 \\
\hline C/1999 S3 (LINEAR) & 82.4 & 1.89 & 1.7235 & $2 / 3 / 11$ & $-126.2,125.0$ & $-19.3,19.2$ & 0.0888 & 0.649 \\
\hline C/1999 F1 (Catalina) & $>10^{3}$ & 5.79 & 1.8790 & $11 / 2 / 11$ & $-123.8,123.9$ & $-15.6,15.6$ & 0.1072 & 0.783 \\
\hline 155P/Shoemaker $3 \bullet$ & 17.1 & 1.81 & 3.1601 & & $-211.6,220.9$ & $-35.5,35.7$ & 0.0769 & 0.562 \\
\hline P/1999 V1 (Catalina) & 16.8 & 2.94 & 3.5145 & & $-340.1,420.2$ & $-68.2,71.1$ & 0.0501 & 0.366 \\
\hline P/1998 U3 (Jager) & 14.9 & 2.13 & 3.6650 & & $-312.8,351.7$ & $-66.9,69.0$ & 0.0114 & 0.083 \\
\hline 140P/Bowell-Skiff • & 16.2 & 1.97 & 3.9698 & & $-222.9,250.6$ & $-37.9,38.5$ & 0.0773 & 0.565 \\
\hline C/2002 B2 (LINEAR) & $>10^{3}$ & 3.84 & 5.6793 & & $-142.6,149.6$ & $-27.6,27.9$ & 0.0430 & 0.314 \\
\hline 134P/Kowal-Vavrova $\bullet$ & 15.6 & 2.58 & 5.7340 & & $-418.2,270.0$ & $-28.7,28.0$ & 0.1205 & 0.881 \\
\hline \multicolumn{9}{|c|}{ Uranus } \\
\hline C/2000 Y2 (Skiff) & $>10^{3}$ & 2.77 & 3.5727 & $7 / 9 / 15$ & $-94.5,95.2$ & & 0.5038 & 4.183 \\
\hline 55P/Tempel-Tuttle & 33.2 & 0.98 & 1.1472 & & $-193.3,207.8$ & & 0.2359 & 1.959 \\
\hline 122P/de Vico & 74.4 & 0.66 & 1.5454 & & $-89.2,89.2$ & & 0.4886 & 4.058 \\
\hline C/1999 XS87 (LINEAR) & 72.8 & 2.77 & 1.6785 & & $-172.1,174.7$ & $-21.5,21.5$ & 0.0952 & 0.791 \\
\hline C/1999 E1 (Li) & 66.4 & 3.92 & 2.3990 & & $-174.8,176.9$ & $-35.1,35.2$ & 0.0056 & 0.047 \\
\hline C/1999 G1 (LINEAR) & 133.5 & 4.04 & 3.7033 & & $-134.8,135.1$ & & 0.3341 & 2.774 \\
\hline C/1998 K2 (LINEAR) & $>10^{3}$ & 2.33 & 4.5063 & & $-134.6,134.0$ & & 0.3053 & 2.535 \\
\hline C/2002 J4 (NEAT) & $>10^{3}$ & 3.63 & 4.5294 & & $-156.6,155.2$ & & 0.1295 & 1.075 \\
\hline C/1998 K3 (LINEAR) & $>10^{3}$ & 3.55 & 5.5976 & & $-151.2,153.3$ & & 0.2261 & 1.878 \\
\hline C/1998 T1 (LINEAR) & $>10^{3}$ & 1.47 & 5.7420 & & $-79.8,80.1$ & & 0.5152 & 4.278 \\
\hline \multicolumn{9}{|c|}{ Neptune } \\
\hline C/1998 M2 (LINEAR) & $>10^{3}$ & 2.73 & 0.6456 & & $-309.9,308.5$ & & 0.3146 & 1.550 \\
\hline C/2001 HT50 (LINEAR-NEAT) & $>10^{3}$ & 2.79 & 3.1194 & & $-212.1,210.8$ & & 0.7954 & 3.920 \\
\hline C/1999 J3 (LINEAR) & $>10^{3}$ & 0.98 & 3.2025 & & $-300.9,300.6$ & & 0.3910 & 1.927 \\
\hline C/1998 Y1 (LINEAR) & 110.0 & 1.75 & 4.0162 & & $-301.9,304.8$ & & 0.5054 & 2.491 \\
\hline C/2000 Y2 (Skiff) & $>10^{3}$ & 2.77 & 5.6308 & & $-278.4,280.9$ & & 0.5691 & 2.805 \\
\hline $\mathrm{C} / 2000 \mathrm{~J} 1$ (Ferris) & 419.5 & 2.54 & 6.0040 & & $-158.1,158.0$ & & 0.8845 & 4.359 \\
\hline
\end{tabular}

